\title{
Calibration and Data Analysis Recommendations for Three-Component Moment Balances
}

\author{
N. Ulbrich ${ }^{\dagger}$ \\ Jacobs Technology Inc., Moffett Field, California 94035 \\ M. Reed $\ddagger$ \\ Aerodyne Industries LLC, Moffett Field, California 94035
}

\begin{abstract}
Fundamental characteristics of design, calibration, and application of threecomponent moment balances are investigated in great detail. These balances are typically used to determine loads on control surfaces, canards, or other parts that are attached to a wind tunnel model. First, three different descriptions of the load state of a moment balance are reviewed. Then, load transformations between different load formats and the combined load diagram for two of the three load components are discussed. An error analysis showed that it is critical to maximize the product of the distance between the bending moment gages and their sensitivities in order to minimize the overall error in the normal force prediction. In addition, it is important to apply a sufficient number of calibration loadings near the first bending moment gage. Then, unwanted near-linear dependencies between the two bending moment gage outputs can be avoided. The error in the bending moment prediction is also investigated that results from the elastic deformation of the metric part of the balance under load. Finally, the application of the Non-Iterative Method to three-component moment balance calibration data is described in order to obtain regression models that can be used to predict loads from measured outputs during a wind tunnel test.
\end{abstract}

\section{Nomenclature}

\footnotetext{
$\dagger$ Aerodynamicist, Jacobs Technology Inc.

† Balance Engineer, Aerodyne Industries LLC.
}

$=$ torsion moment arm

$=$ load vector

$=$ radius of a circle

$=$ output vector
= bending moment arm; not corrected for elastic deformation

$=$ smallest bending moment arm of the balance

$=$ largest bending moment arm of the balance

= bending moment arm; corrected for elastic deformation

$=$ bending moment at the balance moment center

$=$ bending moment at the first bending moment gage

$=$ bending moment at the second bending moment gage

$=$ distance between the second bending moment gage and the balance moment center

$=$ distance between the two bending moment gages

$=$ force caused by dead weight that is used during the manual calibration of a balance

$=$ load vector components of a three-component moment balance

$=$ normal force at the balance moment center

$=$ electrical output of the first bending moment gage

$=$ electrical output of the second bending moment gage

$=$ output vector components of a three-component moment balance

$=$ output of the torsion moment gage

$=$ sensitivity of the bending moment gage

$=$ torsion moment at the balance moment center

$=$ balance axis that is used to describe the bending moment of the balance 
$\delta$

$\triangle B M$

$\triangle B M 1$

$\triangle B M 2$

$\triangle N F$

$\triangle r B M 1$

$\triangle r B M 2$

$\eta_{0}, \eta_{1}, \ldots$

$\lambda_{0}, \lambda_{1}, \ldots$

$\mu_{0}, \mu_{1}, \ldots$

$\nu_{0}, \nu_{1}, \ldots$

$\xi_{0}, \xi_{1}, \ldots$
$=$ balance axis that is used to describe the torsion moment of the balance

$=$ balance axis that is used to describe the normal force of the balance

$=$ angular deflection in radians at a point on the balance where a dead weight is applied

$=$ error of the bending moment if computed using an uncorrected moment arm

$=$ error of the first bending moment

$=$ error of the second bending moment

$=$ error of the normal force

$=$ error of the first bending moment gage output measurement

$=$ error of the second bending moment gage output measurement

$=$ regression coefficients of the normal force

$=$ regression coefficients of the bending moment

$=$ regression coefficients of the torsion moment

$=$ regression coefficients of the first bending moment

$=$ regression coefficients of the second bending moment

\section{Introduction}

Three-component moment balance are often used to measure loads on a subassembly that is attached to the fuselage or wing of a wind tunnel model. The subassembly could be, for example, a control surface, a fin, or a canard. In some sense, a three-component moment balance is a "gaged" model part that allows for the direct measurement of loads that act at or near its attachment point to the wind tunnel model. A wind tunnel user is often interested in measuring the normal force, the bending moment, and the torsion moment that acts on the balance so that the stress in the model's subassembly can be monitored during tests.

Fundamental differences between a three-component moment balance and a six-component primary balance exist that must be taken into consideration during design, calibration, and use of the balance. First, the metric part of a three-component moment balance is flexible, i.e., it elastically deforms under load. It goes from the outer edge of the balance to the first bending moment gage, i.e., the gage that is closest to the outer edge of the balance. This definition results from the fact that the output of the first bending moment gage would remain "constant" and become "unusable" if a hypothetical load would be applied between its location and the balance moment center. The non-metric part of a three-component moment balance, on the other hand, consists of (i) a flexible section with the gages and (ii) a rigid section that is used to attach the balance to the wind tunnel model. The balance is attached to the model using the rigid section of the non-metric part. Therefore, in order to describe balance loads in a coordinate system that can easily be related to the body axis system of the wind tunnel model, the rigid section of the non-metric part to the model must be used to define the balance axis system of the three-component moment balance. This choice also allows for a precise description of the loads during calibration as only the orientation of the rigid section of the non-metric part relative to the gravitational acceleration must be understood assuming dead weights are used for the balance calibration. Table 1 below summarizes the most important differences between a three-component moment balance and a six-component primary balance.

Table 1: Differences between a six-component primary balance and a three-component moment balance.

\begin{tabular}{|c|c|c|}
\hline & Three-Component Moment Balance & Six-Component Primary Balance \\
\hline $\begin{array}{c}\text { Model } \\
\text { Attachment }\end{array}$ & $\begin{array}{l}\underline{\text { rigid section of non-metric part }} \\
\text { attaches to the wind tunnel model }\end{array}$ & $\begin{array}{l}\text { metric part attaches to } \\
\text { the wind tunnel model }\end{array}$ \\
\hline $\begin{array}{c}\text { Elastic Characteristics } \\
\text { of the Metric Part }\end{array}$ & $\begin{array}{c}\text { flexible, i.e., elastically deforms } \\
\text { when balance loads act }\end{array}$ & $\begin{array}{l}\text { rigid, i.e., does not deform } \\
\text { when balance loads act }\end{array}$ \\
\hline $\begin{array}{c}\text { Definition of the } \\
\text { Balance Axis System }\end{array}$ & $\begin{array}{l}\text { orientation of the rigid section of the } \\
\text { non-metric part in space defines axis system }\end{array}$ & $\begin{array}{l}\text { orientation of the metric part } \\
\text { in space defines axis system }\end{array}$ \\
\hline
\end{tabular}

Dead weights are typically used in a balance calibration laboratory to apply calibration loads to a three-component moment balance. This approach limits the type of loadings that can be applied to the balance during calibration. Figure 1 below shows the typical situation during the "manual" loading of a 
three-component moment balance. The dead weight is represented by an "applied" force that is assumed to be parallel to the gravitational acceleration. This applied force acts in the $x-y$ plane of the balance. Therefore, three independent calibration variables, i.e., the applied force $(F)$, the bending moment arm $(a)$, and the torsion moment arm $(b)$, can be varied during calibration. These variables are indirectly connected to the three loads, i.e., the normal force, the bending moment, and the torsion moment, that a wind tunnel user is interested in.

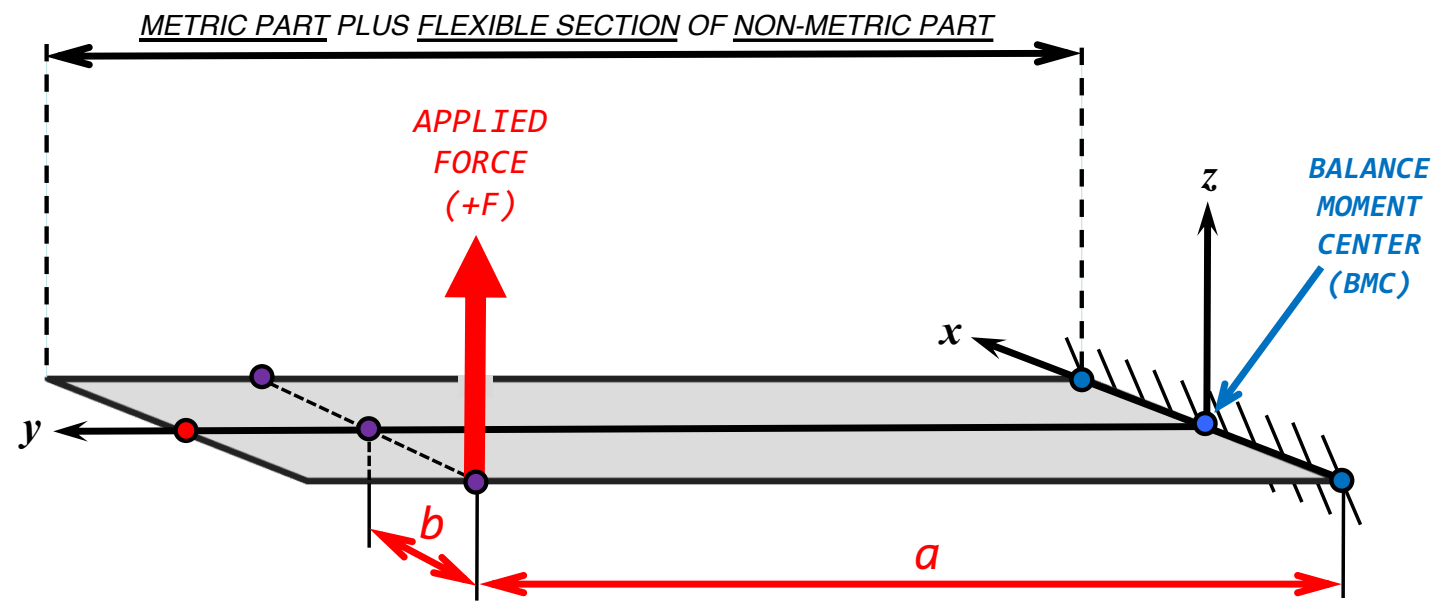

Fig. 1 Manual "loading" of a three-component moment balance that is defined by an applied force, a bending moment arm, and a torsion moment arm.

The authors observed over the years that design, calibration data quality, and load prediction accuracy of three-component balances can vary substantially in a real-world test environment. These observations are not surprising as three-component balances are highly customized one-of-a-kind type load sensors. Nevertheless, the authors have concluded that improvements to design, calibration, and use of three-component moment balances are possible that (i) will reduce balance load prediction errors and (ii) make it easier to apply these load sensors during a wind tunnel test.

An understanding of the suggested improvements requires a more detailed discussion of basic properties of a three-component moment balance. Therefore, fundamental characteristics of this balance type are reviewed in the next section of the paper. These discussions focus primarily on the normal force and the bending moment as the prediction of these loads is interrelated and more complex than the prediction of the torsion moment. Afterwards, improvements to both design and calibration of three-component moment balances are presented. Finally, it is illustrated how the Non-Iterative Method may reliably be used to predict the loads that act on a three-component moment balance.

\section{Balance Characteristics}

\section{A. Load State Descriptions}

In general, an analyst has different variable choices to uniquely describe the "load state" of a threecomponent moment balance. For simplicity, the authors decided to only focus their attention on the normal force and bending moment characteristics as the load prediction accuracy of this pair of loads is interrelated. Therefore, the torsion moment arm and, consequently, the torsion moment itself is assumed to be zero. Then, the "load state" of the balance can be described, for example, by using (i) the applied force and (ii) the bending moment arm. This situation is illustrated in Fig. 2a below. It describes the "load state" from the viewpoint of the "technician" who applies the calibration loads. The balance moment center (BMC) was deliberately placed in Fig. 2a at the junction between "flexible" and "rigid" section of the non-metric part of the balance. This choice makes the three axes of the balance axis system independent of the deformation that the balance experiences under load. 


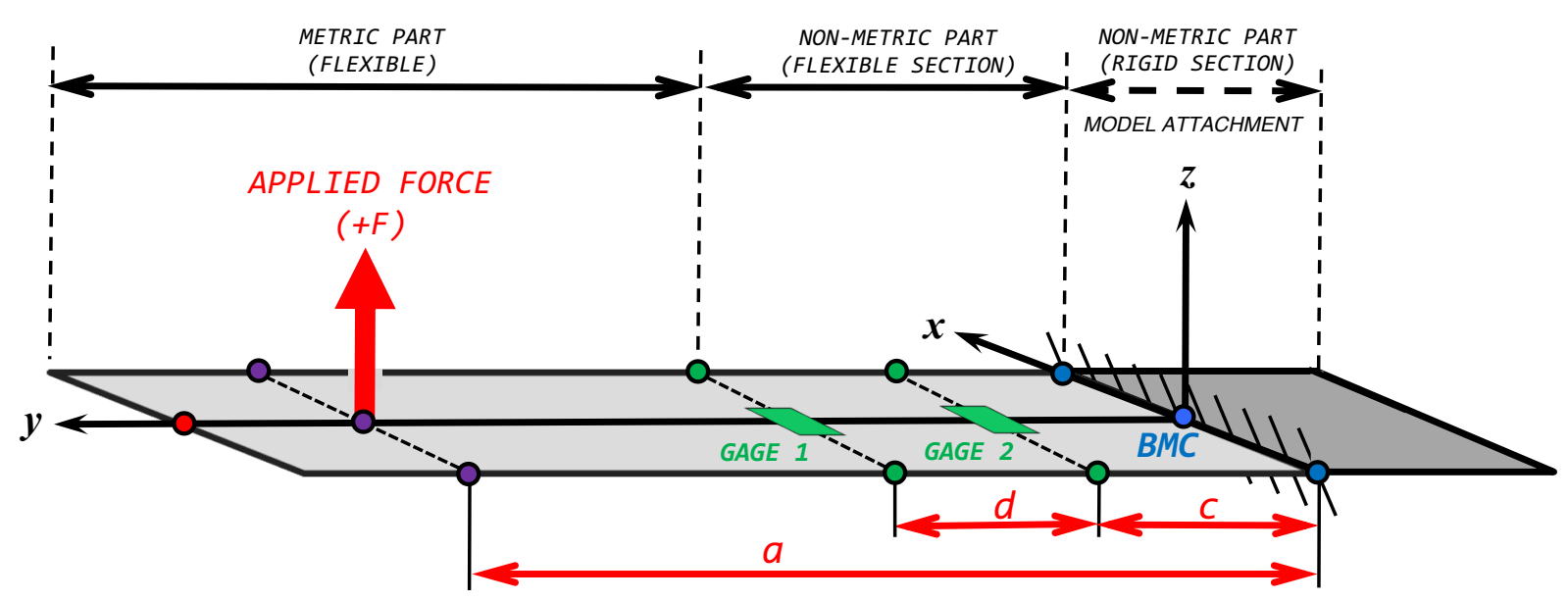

Fig. 2a Description of the "load state" of a three-component moment balance by using the applied force $(F)$ and the bending moment arm $(a)$.

Typical wind tunnel users, on the other hand, prefer a different variable choice in order to describe the "load state" of the balance. They like to describe the "load state" in "direct-read format" using the normal force and the bending moment that act at the BMC. This situation is illustrated in Fig. 2b below.

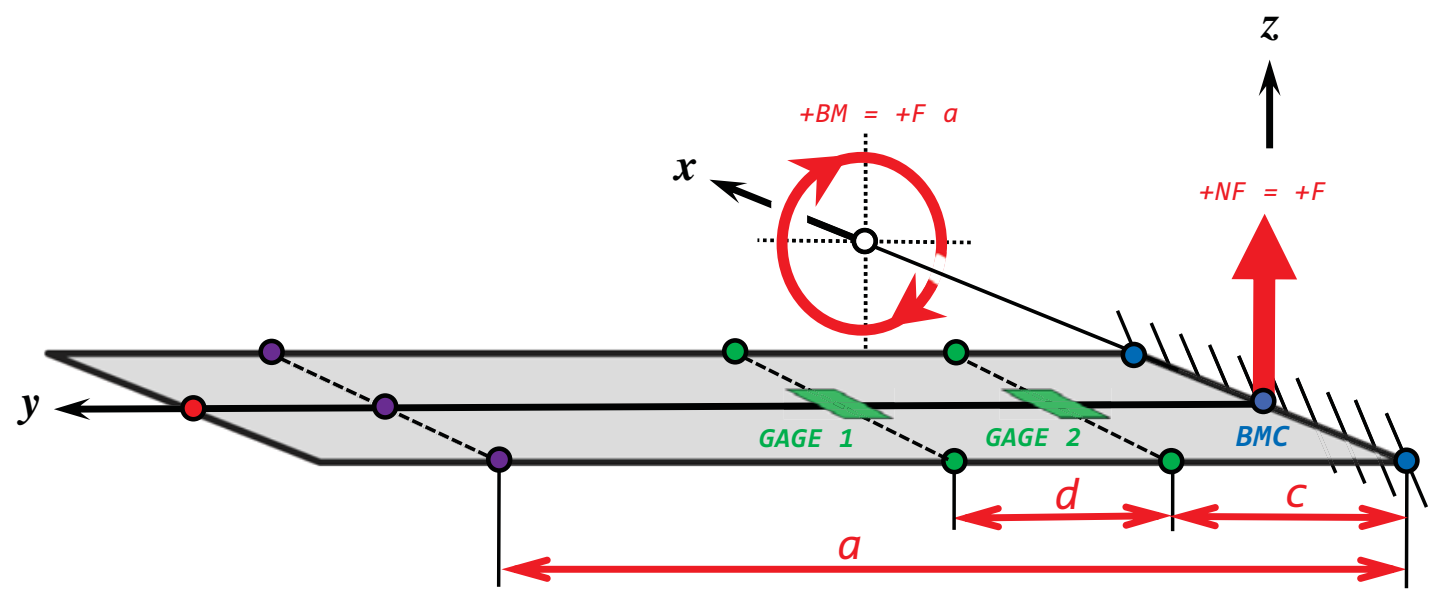

Fig. 2b Description of the "load state" of a three-component moment balance by using the normal force $(N F)$ and the bending moment $(B M)$ at the balance moment center.

A connection between the "load state" given in Fig. 2a and the one given in Fig. 2b exists that can be described by using the following equations:

$$
\begin{aligned}
& N F=F \\
& B M=F \cdot a
\end{aligned}
$$

For completeness, the torsion moment must be included in the description. It is defined as the product between the applied force $(F)$ and the torsion moment arm $(b)$. Then, using the drawing given in Fig. 1, we get the following relationship:

$$
T M=F \cdot b
$$

Finally, balance engineers and some data analysts prefer to describe the "load state" of a moment balance in "moment balance format." Then, the first and second bending moment are used instead of the 
normal force and the bending moment for the description of two of the three load components. The approach has the advantage that (i) the sensitivities of all gages can be defined and (ii) that each applied load is more or less directly proportional to the electrical output of the related gage. This choice is illustrated in Fig. 2c below.

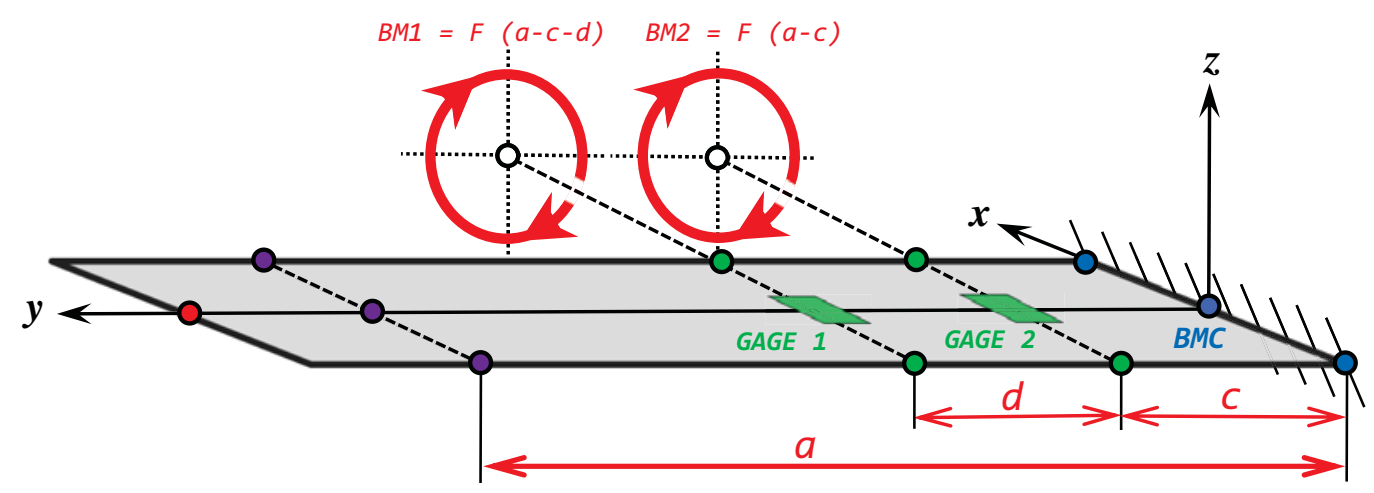

Fig. 2c Description of the "load state" of a three-component moment balance by using the bending moment at gage $1(B M 1)$ and the bending moment at gage $2(B M 2)$.

The first and second bending moments can be obtained by multiplying the applied force with the distances to the centers of the first and second bending moment gage. Then, the two bending moments can be computed by using the following expressions:

$$
\begin{aligned}
& B M 1=F \cdot[a-c-d] \quad \Longrightarrow \quad \text { proportional to } r B M 1 \\
& B M 2=F \cdot[a-c] \quad \Longrightarrow \text { proportional to } r B M 2
\end{aligned}
$$

The torsion moment, of course, is not influenced by the new bending moment definitions. It is still defined as the product between the applied force $(F)$ and the torsion moment arm $(b)$ :

$$
T M=F \cdot b \Longrightarrow \text { proportional to } r T M
$$

Load transformation equations between the normal force and the bending moment and the first and second bending moments at the gages can easily be derived by using Eqs. (1a) and (1b) to replace the applied load $(F)$ and the bending moment arm $(a)$ in Eqs. $(2 a)$ and (2b) (see also Ref. [1] for a description of load transformations for a six-component moment balance). Then, after some algebra, we get the following transformation equations that relate the load pair $N F \& B M$ to the alternate load pair $B M 1 \& B M 2$ :

$$
\begin{aligned}
& N F=(B M 2-B M 1) / d \\
& B M=B M 2+(B M 2-B M 1) \cdot \frac{c}{d}
\end{aligned}
$$

Important improvements related to the calibration of a three-component moment balance are presented in a later section of the paper. These improvements can better be understood if the combined load diagrams of the normal force and the bending moments of the balance are discussed (see Ref. [2] for more details about combined load diagrams). This visualization of the balance load envelope is described in the next section of the paper.

\section{B. Combined Load Diagram}

The combined load diagram of a pair of balance loads is a useful tool to better understand loadings that are applied to the balance during calibration. Therefore, it was decided to prepare the combined load diagram for the load pairs that are related to the two bending moment gage measurements. First, the combined load diagram is developed in "direct-read" format. In that case, the normal force and the bending 
moment at the balance moment center describe the "load state" of the balance. Figure 3a below shows a typical loading situation in "direct-read" format.

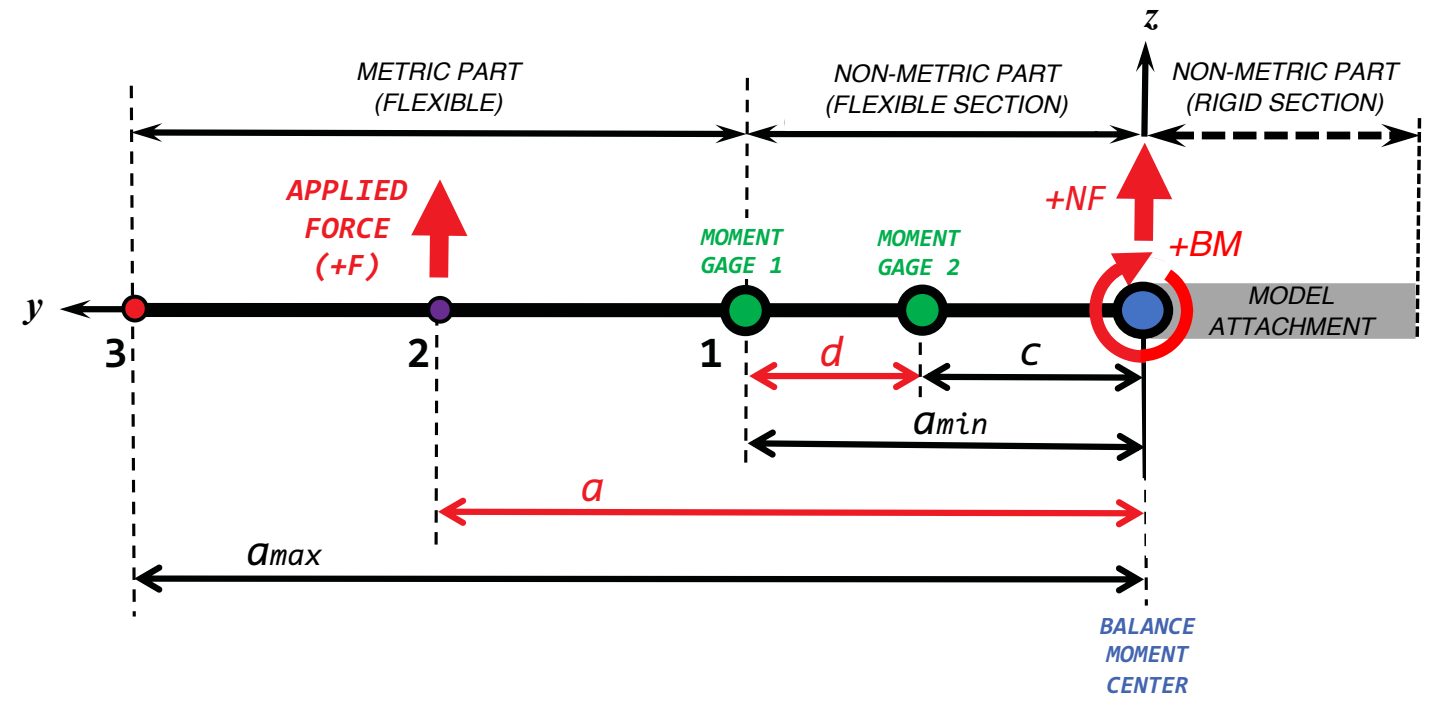

Fig. 3a Description of the loads of a three-component moment balance in "direct-read" format.

Loads can only be applied on the metric part of the balance. Related load points are located in Fig. 3a between point 1 and point 3 . Now, after analyzing all possible load cases, the combined load diagram for the normal force and bending moment is obtained (see Fig. 3b below).

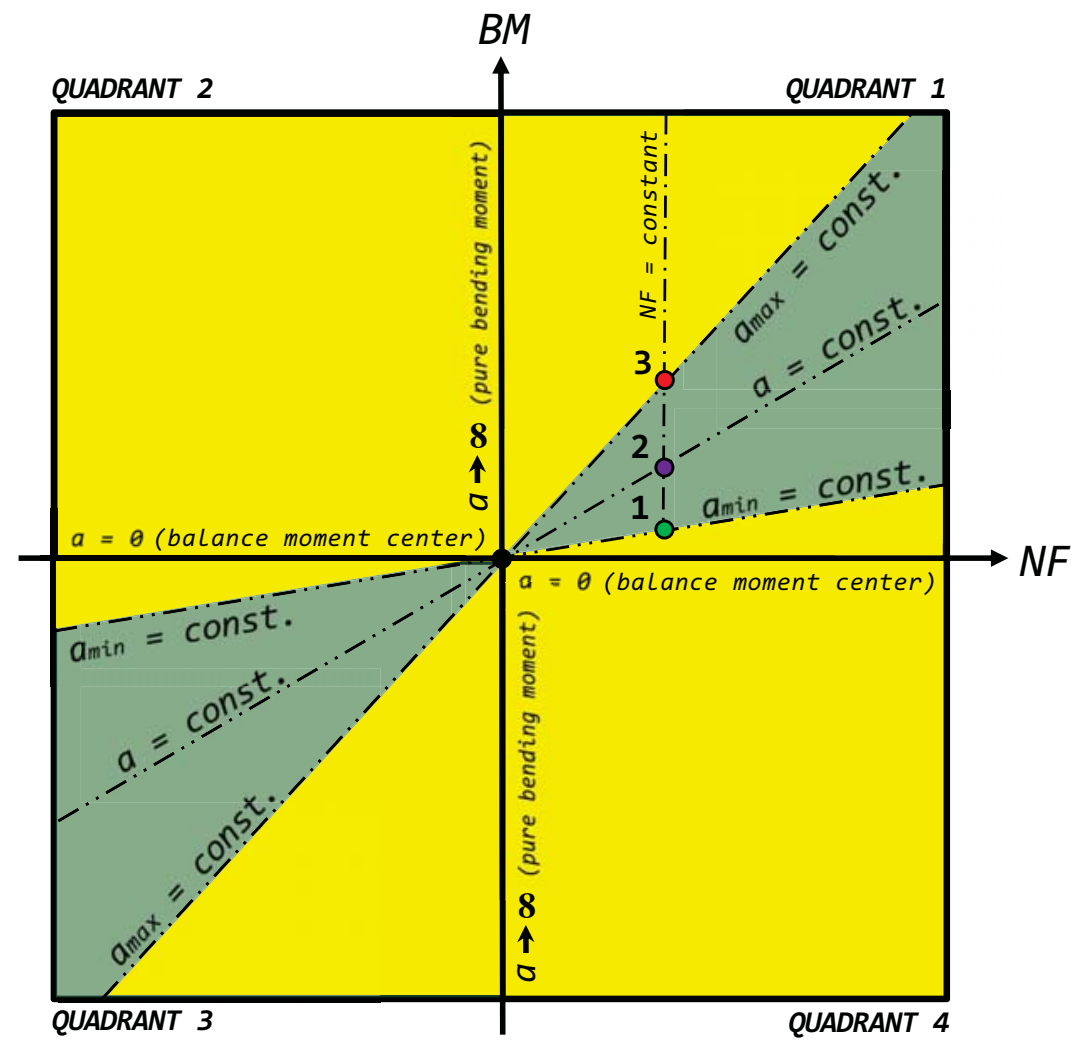

Fig. 3b Combined load diagram of a three-component moment balance in "direct-read" format. 
Loads can only appear in the dark green regions that are shown in Fig. 3b assuming that a single dead weight is applied at the load point during the calibration of the balance. In the next step, the combined load diagram is developed in "moment balance" format. In that case, the first and second bending moment at the gage locations describe the "load state" of the balance. Figure 4a below shows a typical loading situation in "moment balance" format.

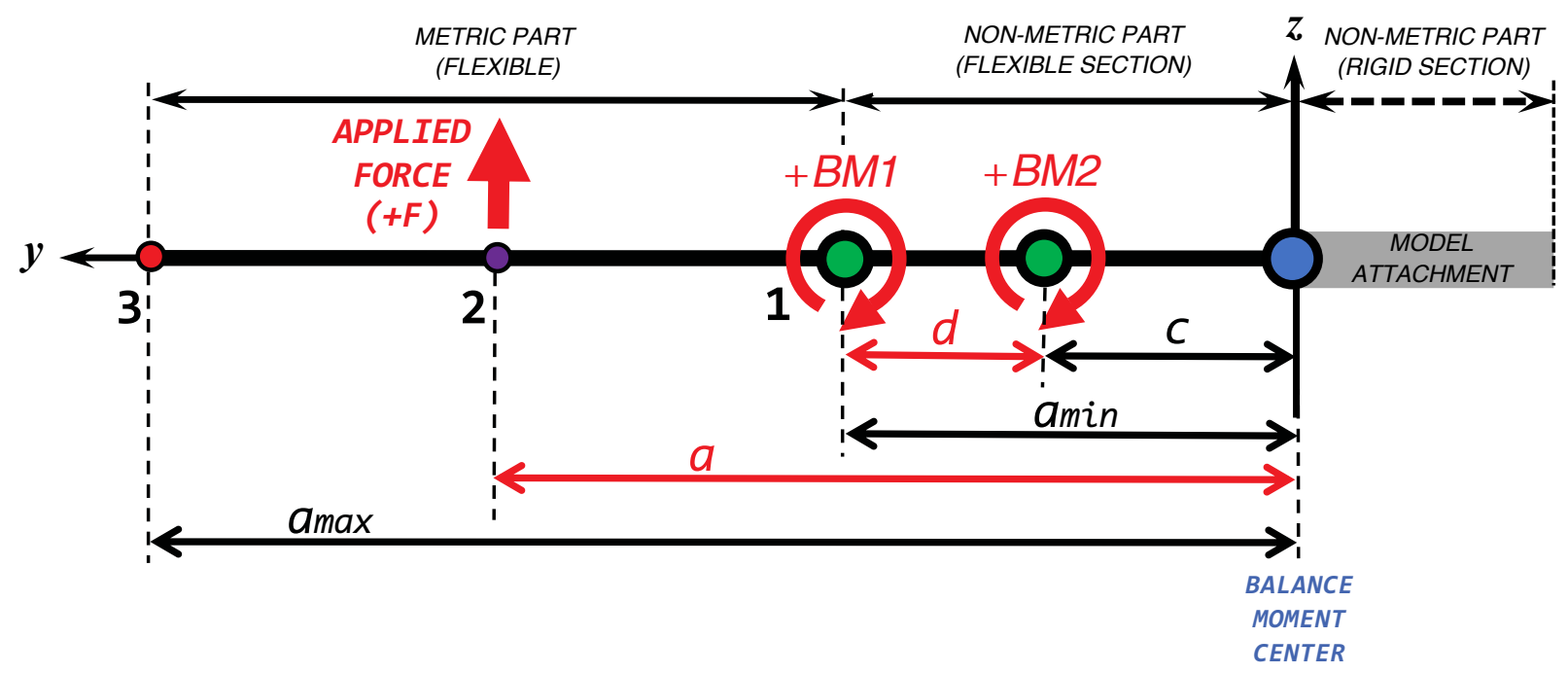

Fig. 4a Description of the loads of a three-component moment balance in "moment balance" format.

The corresponding combined load diagram can be obtained after analyzing all typical load cases. It is shown in Fig. 4b below.

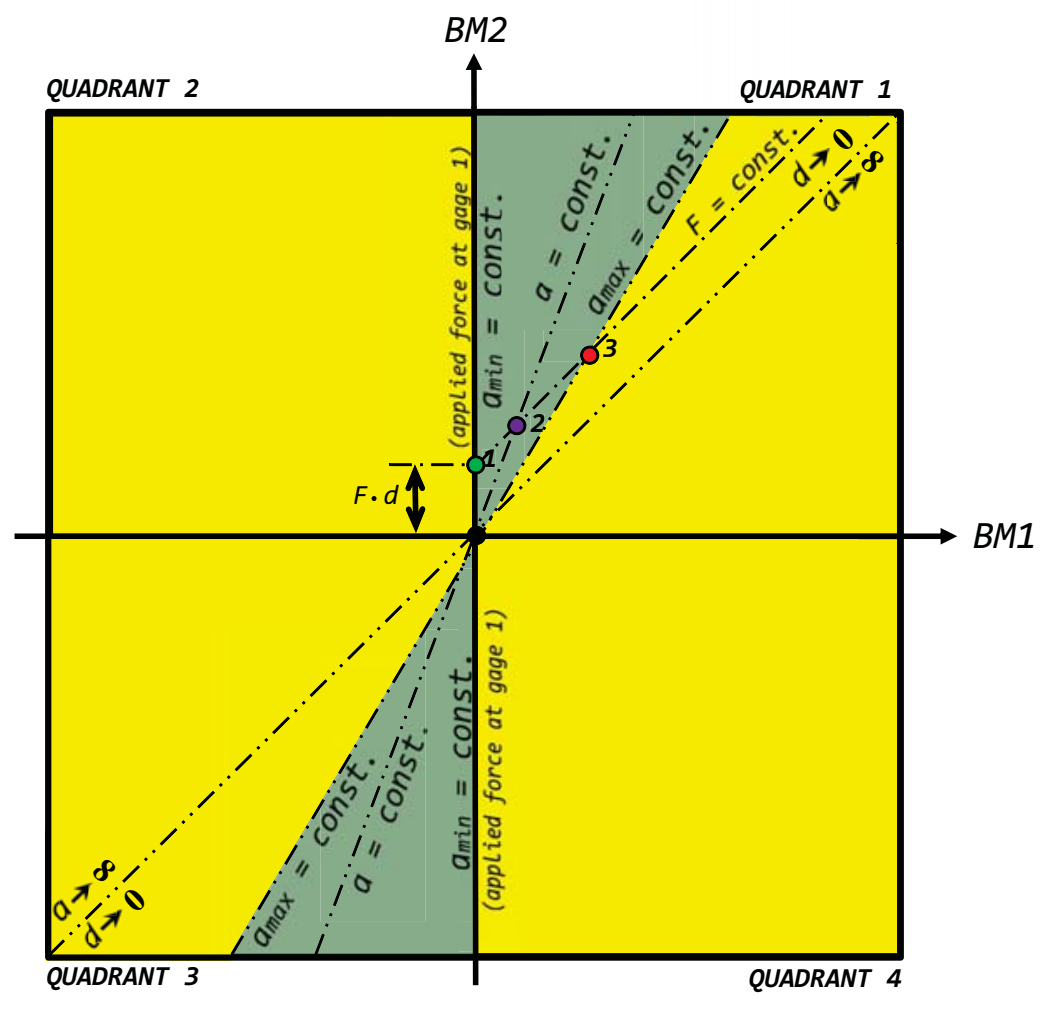

Fig. 4b Combined load diagram of a three-component moment balance in "moment balance" format. 
Again, loads can only appear in the dark green regions that are shown in Fig. 4b assuming that a single dead weight is applied at the load point during the calibration of the balance. The principle diagonal of the first and third quadrant has an important characteristic: it is the location of load points where the two bending moments are identical. This situation can be achieved by either making the distance between the two bending moment gages zero or by using a bending moment arm that is at "infinity". Consequences of these two conclusions will be discussed in more detail in the next section of the paper.

\section{Design and Calibration Recommendations}

\section{A. Gage Placement and Sensitivity Selection}

In the previous section it was mentioned that the first and second bending moments are identical when the distance between the gages approaches zero. Then, the two bending moment gage outputs are no longer independent. Consequently, the balance would only have two instead of the required three independent electrical output measurements that are needed to predict the normal force, the bending moment, and the torsion moment.

An estimate of the upper bound of the error of the normal force as a function of the errors of the two bending moments can be used to illustrate this issues. A "conservative" upper bound of the error of the normal force can be obtained by taking the absolute values of both sides of Eq. (3a). Then, we get:

$$
\text { conservative error estimate } \Longrightarrow|\Delta N F| \leq\{|\Delta B M 2|+|\Delta B M 1|\} / d
$$

In addition, it is known that the gage sensitivity may be used to directly relate the load to the measured output if the balance loads are given in moment balance format. Then, we get the following relationships for the two bending moments:

$$
\begin{aligned}
& B M 1 \approx \underbrace{\frac{1}{\partial r B M 1 / \partial B M 1}}_{\text {gage sensitivity }} \cdot r B M 1 \\
& B M 2 \approx \underbrace{\frac{1}{\partial r B M 2 / \partial B M 2}}_{\text {gage sensitivity }} \cdot r B M 2
\end{aligned}
$$

The bending moment gages of three-component moment balances are typically selected to have identical sensitivities. Therefore, we can make the following simplification:

$$
S_{B M} \approx \partial r B M 1 / \partial B M 1 \approx \partial r B M 2 / \partial B M 2
$$

Then, after using Eqs. $(5 a)$ and $(5 b)$ in combination with Eq. (6), we get the following approximations of the errors of the two bending moments:

$$
\begin{aligned}
\Delta B M 1 & \approx \frac{\Delta r B M 1}{S_{B M}} \\
\Delta B M 2 & \approx \frac{\Delta r B M 2}{S_{B M}}
\end{aligned}
$$

Finally, after using the right-hand sides of Eqs. $(7 a)$ and $(7 b)$ to replace the bending moment errors in Eq. (4) above, we get the following estimate of the error of the normal force:

$$
\text { conservative error estimate } \Longrightarrow|\Delta N F| \leq \frac{|\Delta r B M 1|+|\Delta r B M 2|}{d \cdot S_{B M}}
$$

The product of (i) the distance between the bending moment gages and (ii) the sensitivity of the bending moment gages is in the denominator of the right-hand side of Eq. (8). Therefore, it is concluded that this product must be maximized within given geometric constraints of the three-component moment balance in 
order to minimize the error in the prediction of the normal force of the balance. This important conclusion can be summarized as follows:

\section{BALANCE DESIGN RECOMMENDATION}

The product of (i) the distance between the two bending moment gages and (ii) the sensitivity of the bending moment gages must be maximized in order to minimize the overall error in the normal force prediction.

The authors' experience has shown that the distance between the bending moment gages also indirectly influences the reliability of the regression models of the loads. Small distances may create bending moment gage output sets with hidden linear or near-linear dependencies unless a significant number of calibration loadings is applied in the vicinity of the first bending moment gage. This calibration load schedule design issue is discussed in more detail in the next section of the paper.

\section{B. Calibration Load Schedule Improvement}

It was shown in Fig. 4b that either an infinitely large bending moment arm or an infinitesimally small distance between the bending moment gages makes the two bending moments and related bending moment gage outputs identical. This characteristic can become a serious problem if a very large bending moment arm is used during the calibration or a balance with a very small distance between the bending moment gages is calibrated. The ratio between the two bending moments can be used to illustrate this problem. Then, after dividing both sides of Eq. (2a) by both sides of Eq. (2b), we get the following equation for the ratio of the bending moments:

$$
\frac{B M 1}{B M 2}=\frac{a-c-d}{a-c}=1-\frac{1}{(a-c) / d}
$$

It is also reasonable to assume that the bending moment arm is significantly larger than the distance between the second bending moment gage and the balance moment center. Then, we get the following approximation of the ratio of the bending moments:

$$
a \gg c \Longrightarrow \frac{B M 1}{B M 2} \approx 1-\frac{1}{a / d}
$$

Consequently, the ratio $B M 1 / B M 2$ between the two bending moments will be close to the unwanted value of one whenever the ratio $a / d$ between the bending moment arm and the gage distance is large. The same situation will be observed for the ratio $r B M 1 / r B M 2$ of the electrical outputs of the bending moment gages as $r B M 1$ is proportional to $B M 1$ and $r B M 2$ is proportional to $B M 2$. This conclusion can be summarized as follows:

$$
a / d \gg 1 \Longrightarrow \frac{B M 1}{B M 2} \approx 1 \quad \Longrightarrow \quad B M 1=B M 2 \quad \text { or } \quad r B M 1=r B M 2
$$

Many calibration load schedules of real-world three-component moment balances have the characteristic that the smallest value of the ratio $a / d$ is between four and six. These data sets make it difficult for the regression analysis process to come up with robust regression models of the loads as the two bending moment gage outputs "appear" to be almost linear related. This data analysis issue can easily be addressed by taking a significant number of calibration points near the first bending moment gage. Consequently, the following load schedule design recommendation can be made:

American Institute of Aeronautics and Astronautics 


\section{CALIBRATION LOAD SCHEDULE DESIGN RECOMMENDATION}

Large ratios between (i) the bending moment arm and (ii) the bending moment gage distance generate electrical outputs of the two bending moment gages that are almost identical. Therefore, its is absolutely critical to include a significant number of calibration points in the load schedule that are "close" to the first bending moment gage so that the electrical outputs of the calibration data do not "appear" to be linearly related.

It was mentioned in Table 1 above that both the metric and the gaged part of the non-metric part of a three-component moment balance are subject to elastic deformation. Therefore, depending on the degree of deformation, it may be necessary to apply a moment arm correction when the bending moments are computed. This correction is discussed in the next section of the paper.

\section{Bending Moment Arm Correction}

The metric part and parts of the non-metric part of a three-component moment balance deform when the balance sees a load. In other words, the bending moment arm of the resultant force is not constant. Therefore, the authors developed a moment arm correction for the balance calibration data that takes the deflection of the balance under load into account. Figure 5a below shows the deflection of the balance in a loaded state.

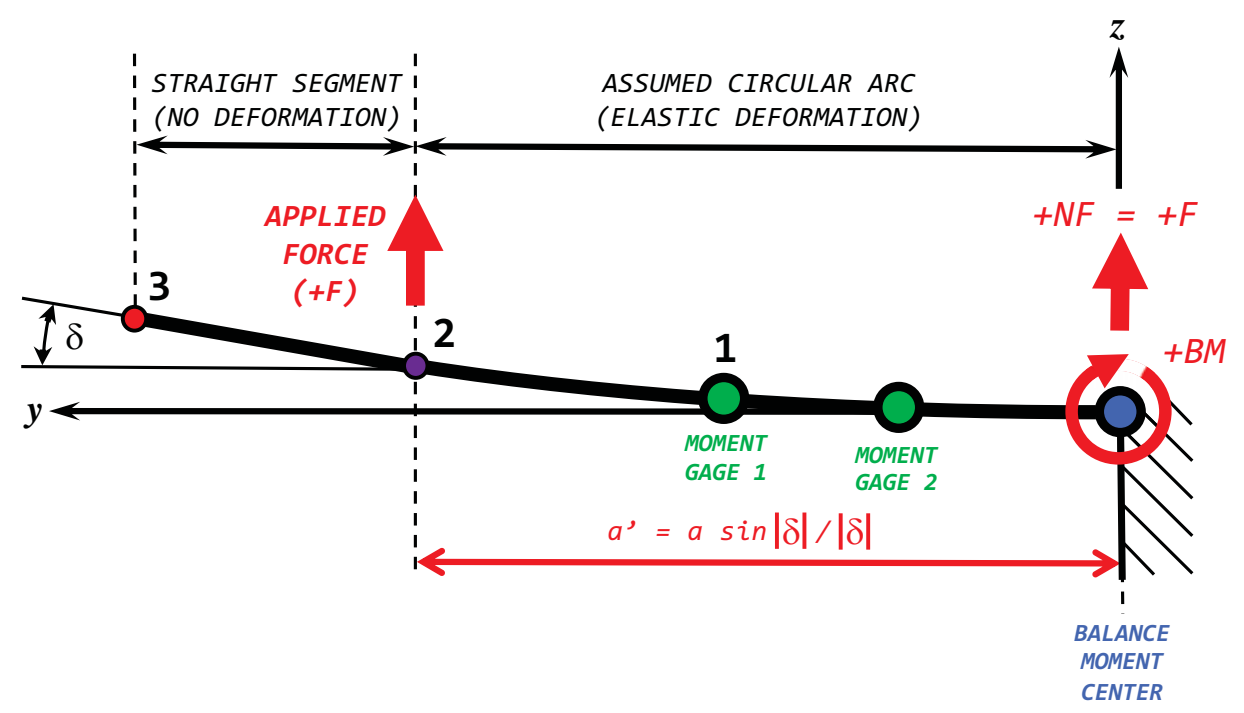

Fig. 5a Moment arm correction for a "loaded" three-component moment balance.

The moment arm correction can be computed if the deflection angle $\delta$ is measured at the location where a calibration load is applied. In addition, the assumption is made that the shape of the deflection curve between the balance moment center and the load point is a circular arc. Figure 5b below shows the connection between the basic geometric parameters that results from this assumption. 


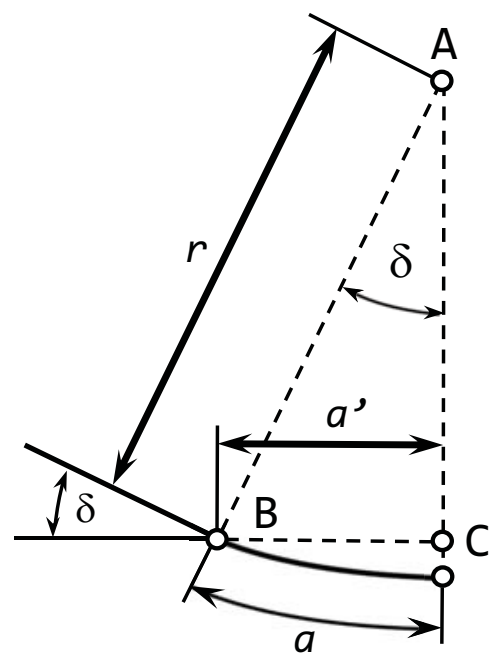

Fig. 5b Geometric relationship between uncorrected moment arm $(a)$ and corrected moment arm $\left(a^{\prime}\right)$.

Now, knowing that the ratio between the arc $(a)$ and the circumference of the circle $(2 \pi r)$ equals the ratio between the deflection angle $(\delta)$ and $2 \pi$, and, solving the resulting equation for the radius of the circle, we get:

$$
\frac{a}{2 \pi r}=\frac{\delta}{2 \pi} \Longrightarrow r=\frac{a}{\delta}
$$

In addition, "ABC" in Fig. 5b above is a right triangle. Then, the "sine" of the deflection angle can be computed as a function of the corrected moment arm $\left(a^{\prime}\right)$ and the radius $(r)$. Now, after solving the resulting equation again for the radius, we get:

$$
\sin (\delta)=\frac{a^{\prime}}{r} \Longrightarrow r=\frac{a^{\prime}}{\sin (\delta)}
$$

Finally, after setting the right-hand side of Eq. (11a) equal to the right-hand side of Eq. (11b) and solving the resulting equation for the corrected moment arm $\left(a^{\prime}\right)$, we get:

$$
a^{\prime}=a \cdot \frac{\sin (\delta)}{\delta}
$$

The error of the computed bending moment can be obtained by taking the difference of the bending moments for the two bending moment arm choices where $B M$ is the uncorrected bending moment and $B M^{\prime}$ is the corrected bending moment. Then, using the bending moment definition given in Eq. (1b), we get:

$$
\Delta B M=B M-B M^{\prime}=\overbrace{F \cdot a}^{\text {uncorrected }}-\overbrace{F \cdot \underbrace{a \cdot \sin (\delta) / \delta}_{a^{\prime}}}^{\text {corrected }}
$$

The right-hand side of Eq. (13a) can be simplified for improved clarity. Then, we get:

$$
\Delta B M=F \cdot a \cdot\{1-\sin (\delta) / \delta\}=B M \cdot\{1-\sin (\delta) / \delta\}
$$

Consequently, after rearranging Eq. (13b) and multiplying the result by $100 \%$, the relative error of the bending moment due to deflection can be computed in percent as follows:

$$
\left\{\frac{\Delta B M}{B M}\right\}_{\%}=\{1-\sin (\delta) / \delta\} \cdot 100 \%
$$


It is useful to evaluate the error estimate defined in Eq. (14) for a family of deflection angles. The authors decided to use the following deflection angle set: $2 \mathrm{deg}, 5 \mathrm{deg}, 10 \mathrm{deg}$, and $15 \mathrm{deg}$. Table 2 below lists the result of the error estimate of the bending moment for the four chosen deflection angles.

Table 2: Bending moment error as a function of balance deflection.

\begin{tabular}{|c|c|c|}
\hline$\delta \equiv$ deflection angle & $\sin |\delta| /|\delta|$ & $(\Delta B M / B M)_{\%}$ \\
\hline \hline $0.03491 \mathrm{rad} \equiv 2[\mathrm{deg}]$ & 0.9998 & $0.02 \%$ \\
\hline $0.08727 \mathrm{rad} \equiv 5[\mathrm{deg}]$ & 0.9987 & $0.13 \%$ \\
\hline $0.17453 \mathrm{rad} \equiv 10[\mathrm{deg}]$ & 0.9949 & $0.51 \%$ \\
\hline $0.26180 \mathrm{rad} \equiv 15[\mathrm{deg}]$ & 0.9886 & $1.14 \%$ \\
\hline
\end{tabular}

It is observed that the bending moment correction may not have to be applied if the deflection angle is less equal $5[\mathrm{deg}]$ because the difference between the uncorrected and corrected bending moments is on the order of $0.1 \%$ or less. The two larger deflection angles, on the other hand, are causing differences that are on the order of $1.0 \%$. Therefore, it is concluded that the bending moment correction may have to be applied if (i) the deflection angle exceeds the threshold of 5 [deg] and (ii) the user of a three-component moment balance has very high accuracy requirements. - The regression analysis of the balance calibration data is discussed in more detail in the next section of the paper.

\section{Data Analysis}

Different methods are used in the aerospace testing community to predict balance loads from strain-gage outputs during a wind tunnel test. Some analysts prefer to apply the Iterative Method (see, e.g., Ref. [3] for a description of the method). This approach fits outputs as a function of loads that were applied during a balance calibration. Afterwards, a load iteration scheme is constructed from the regression analysis results so that loads can be predicted from outputs during a wind tunnel test. The Non-Iterative Method may also be used for the prediction of balance loads (see Ref. [4] for more detail). This alternate approach is much simpler to apply than the Iterative Method. It directly fits calibration loads as a function of measured outputs. The accuracy of both methods is similar as long as hidden linear or near-linear dependencies between terms of the regression model of the balance calibration data are avoided. Therefore, the authors decided to select the Non-Iterative Method for the prediction of the loads of a three-component moment balance.

The application of the Non-Iterative Method to a three-component moment balance needs to be explained in more detail (see again Ref. [4] for a detailed discussion of this approach). In this case, loads are directly fitted as a function of the measured gage outputs. It is useful to introduce the concept of an "output space" and a "load space" during the discussion of the Non-Iterative Method (see also Ref. [5]). The NonIterative Method essentially creates a "unique" mapping between the two spaces. This idea is summarized in Fig. 6 below.

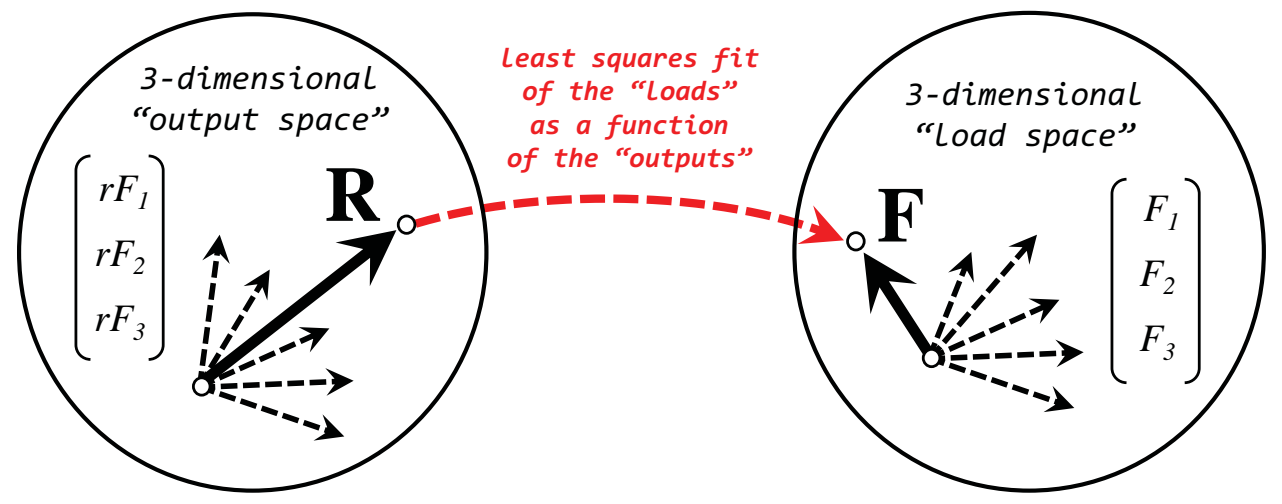

Fig. 6 Definition of the "load space" and "output space" of a three-component moment balance. 
Each point in the "output space" describes a specific "load state" of the balance. The same "load state" is described by a point in the "load space". In our case, points in the "output space" are described as three-dimensional vectors. The vector components are the two bending moment gage outputs and the torsion moment gage output. This can be described in vector format as follows:

$$
\text { Output Vector } \Longrightarrow \mathbf{R}=\left[\begin{array}{c}
r F_{1} \\
r F_{2} \\
r F_{3}
\end{array}\right]=\left[\begin{array}{c}
r B M 1 \\
r B M 2 \\
r T M
\end{array}\right]
$$

Two options exist to describe a point in the "load space" that depend on the chosen load format of the balance. It is possible to describe the loads in direct-read format. Then, the load vector has the following definition:

$$
\text { Load Vector (Option 1) } \Longrightarrow \text { direct-read format } \Longrightarrow \mathbf{F}=\left[\begin{array}{c}
F_{1} \\
F_{2} \\
F_{3}
\end{array}\right]=\left[\begin{array}{c}
N F \\
B M \\
T M
\end{array}\right]
$$

It is also possible to describe the load vector in moment balance format. Then, the load vector has the following definition:

$$
\text { Load Vector (Option 2) } \Longrightarrow \text { moment balance format } \Longrightarrow \mathbf{F}=\left[\begin{array}{c}
F_{1} \\
F_{2} \\
F_{3}
\end{array}\right]=\left[\begin{array}{c}
B M 1 \\
B M 2 \\
T M
\end{array}\right]
$$

Regression models need to be developed so that the measured electrical outputs of the two bending moments and the torsion moment gage can be used to predict the balance loads. The selection of the regression model terms of the three load components depends on the calibration load schedule. In theory, a total of ten regression model terms are supported by a typical load schedule of a three-component moment balance as the torsion moment and the bending moments are often applied simultaneously. The ten terms of the resulting second order Taylor Series approximation of the balance loads are listed in Table 3 below.

Table 3: Regression model terms for the loads of a three-component moment balance.

\begin{tabular}{|c|c|}
\hline Type & List of Regression Model Terms \\
\hline \hline constant $(1)$ & Intercept \\
\hline linear $(3)$ & $(r B M 1),(r B M 2),(r T M)$ \\
\hline quadratic $(3)$ & $(r B M 1)^{2},(r B M 2)^{2},(r T M)^{2}$ \\
\hline cross-product $(3)$ & $(r B M 1) \cdot(r B M 2),(r B M 1) \cdot(r T M),(r B M 2) \cdot(r T M)$ \\
\hline
\end{tabular}

First, let us assume that loads are described in direct-read format. Then, the regression models of the normal force and the bending moment are defined by the following equations:

$$
\begin{aligned}
N F=\eta_{0} & +\underbrace{\eta_{1} \cdot(r B M 1)+\eta_{2} \cdot(r B M 2)+\eta_{3} \cdot(r T M)}_{\text {linear terms }} \\
& +\underbrace{\eta_{4} \cdot(r B M 1)^{2}+\eta_{5} \cdot(r B M 2)^{2}+\eta_{6} \cdot(r T M)^{2}}_{\text {quadratic terms }} \\
& +\underbrace{\eta_{7} \cdot(r B M 1) \cdot(r B M 2)+\eta_{8} \cdot(r B M 1) \cdot(r T M)+\eta_{9} \cdot(r B M 2) \cdot(r T M)}_{\text {cross-product terms }} \\
B M=\lambda_{0} & +\underbrace{\lambda_{1} \cdot(r B M 1)+\lambda_{2} \cdot(r B M 2)+\lambda_{3} \cdot(r T M)}_{\text {quadratic terms }} \\
& +\underbrace{\lambda_{4} \cdot(r B M 1)^{2}+\lambda_{5} \cdot(r B M 2)^{2}+\lambda_{6} \cdot(r T M)^{2}}_{\text {cross-product terms }} \\
& +\underbrace{\lambda_{7} \cdot(r B M 1) \cdot(r B M 2)+\lambda_{8} \cdot(r B M 1) \cdot(r T M)+\lambda_{9} \cdot(r B M 2) \cdot(r T M)}
\end{aligned}
$$

American Institute of Aeronautics and Astronautics 
Alternatively, loads may be expressed in moment balance format. This approach has the advantage that the sensitivities of all balance gages are defined. In addition, troubleshooting is simplified because the bending moment gage outputs are directly proportional to the applied first and second bending moment. Then, the regression models of the first and second bending moment can be defined as follows:

$$
\begin{aligned}
B M 1=\nu_{0} & +\underbrace{\nu_{1} \cdot(r B M 1)+\nu_{2} \cdot(r B M 2)+\nu_{3} \cdot(r T M)}_{\text {linear terms }} \\
& +\underbrace{\nu_{4} \cdot(r B M 1)^{2}+\nu_{5} \cdot(r B M 2)^{2}+\nu_{6} \cdot(r T M)^{2}}_{\text {quadratic terms }} \\
& +\underbrace{\nu_{7} \cdot(r B M 1) \cdot(r B M 2)+\nu_{8} \cdot(r B M 1) \cdot(r T M)+\nu_{9} \cdot(r B M 2) \cdot(r T M)}_{\text {cross-product terms }} \\
& +\underbrace{\xi_{4} \cdot(r B M 1)^{2}+\xi_{5} \cdot(r B M 2)^{2}+\xi_{6} \cdot(r T M)^{2}}_{\text {linear terms }} \\
& +\underbrace{\xi_{7} \cdot(r B M 1) \cdot(r B M 2)+\xi_{8} \cdot(r B M 1) \cdot(r T M)+\xi_{9} \cdot(r B M 2) \cdot(r T M)}_{\text {quadratic terms }}
\end{aligned}
$$

Finally, the regression model of the torsion moment is defined by the following equation:

$$
\begin{aligned}
T M=\mu_{0} & +\underbrace{\mu_{1} \cdot(r B M 1)+\mu_{2} \cdot(r B M 2)+\mu_{3} \cdot(r T M)}_{\text {linear terms }} \\
& +\underbrace{\mu_{4} \cdot(r B M 1)^{2}+\mu_{5} \cdot(r B M 2)^{2}+\mu_{6} \cdot(r T M)^{2}}_{\text {quadratic terms }} \\
& +\underbrace{\mu_{7} \cdot(r B M 1) \cdot(r B M 2)+\mu_{8} \cdot(r B M 1) \cdot(r T M)+\mu_{9} \cdot(r B M 2) \cdot(r T M)}_{\text {cross-product terms }}
\end{aligned}
$$

A superficial application of the regression models defined in Eqs. (17a) to (19) must be avoided at all cost as only a subset of the ten terms may truly be supported by the given calibration data. Therefore, it is critical to screen the final set of regression model terms for hidden linear or massive near-linear dependencies using a metric like the "variance inflation factor" as, for example, the outputs of the two bending moment gages can easily "appear" linearly dependent if none or too few calibration loadings are applied near the first bending moment gage (see Ref. [7] for a detailed discussion of the metric). In addition, the term $(r T M)^{2}$ is often not supported by a given "real-world" calibration data set as only three closely spaced moment arm positions may be available to apply the torsion moment.

The accuracy of the balance load prediction can often be increased if a tare load iteration is performed in combination with the application of the Non-Iterative Method. Then, all calibration loads are expressed relative to the absolute load datum of zero load (see Ref. [6] for more details). The regression coefficients are known after performing the least squares fit of the data. Again, the Non-Iterative Method has the advantage that it leads to three explicit equations for the loads in either direct-read or moment balance format that can easily be implemented and evaluated in the data system of a wind tunnel test.

\section{Summary}

Fundamental characteristics of design, calibration, and use of three-component moment balances were investigated in great detail. First, basic features of this balance type were reviewed that included a discussion of different load formats and the definition of the combined load diagram. Afterwards, balance design and calibration recommendations were made. First, the authors recommend to maximize the product between (i) the bending moment gage distance and (ii) the bending moment gage sensitivity in order to minimize 
the load prediction error for the normal force. A balance user needs to work closely with the manufacturer of the balance to achieve this goal if the accuracy of the normal force prediction is critial for a given test objective. In addition, the authors suggest to apply a significant number of calibration loads in the vicinity of the first bending moment gage in order to avoid a situation when the two bending moment gage outputs of the balance "appear" to be linearly related. A correction formula for the bending moment arm was also developed that takes the deformation of the metric part of the balance under load into account. Finally, basic elements of the use of the Non-Iterative Method for the prediction of the loads of a three-component moment balance were presented.

\section{Acknowledgements}

The author wants to thank Tom Volden of Jacobs Technology and Bob Gisler of NASA Ames Research Center for their critical and constructive review of the final manuscript. The work reported in this paper was partially supported by the Wind Tunnel Division at NASA Ames Research Center under contract NNA16BD26C.

\section{References}

[1] Ulbrich, N., and Bader, J., "Analysis of Sting Balance Calibration Data Using Optimized Regression Models," AIAA 2009-5372, paper presented at the 45th AIAA/ASME/SAE/ASEE Joint Propulsion Conference, Denver, Colorado, August 2009.

[2] Ulbrich, N., "Combined Load Diagram for a Wind Tunnel Strain-Gage Balance," AIAA 2010-4203, paper presented at the 27th AIAA Aerodynamic Measurement Technology and Ground Testing Conference, Chicago, Illinois, June/July 2010.

[3] AIAA/GTTC Internal Balance Technology Working Group, Recommended Practice, Calibration and Use of Internal Strain-Gage Balances with Application to Wind Tunnel Testing, AIAA R-091-2003, American Institute of Aeronautics and Astronautics, Reston, Virginia, 2003.

[4] Ulbrich, N., "Comparison of Iterative and Non-Iterative Strain-Gage Balance Load Calculation Methods," AIAA 2010-4202, paper presented at the 27th AIAA Aerodynamic Measurement Technology and Ground Testing Conference, Chicago, Illinois, June/July 2010.

[5] Ulbrich, N., "Assessment of the Uniqueness of Strain-Gage Balance Load Predictions," AIAA 20164157, paper presented at the 32nd AIAA Aerodynamic Measurement Technology and Ground Testing Conference, Washington, D.C., June 2016.

[6] Ulbrich, N., "A Universal Tare Load Prediction Algorithm for Strain-Gage Balance Calibration Data Analysis," AIAA 2011-6090, paper presented at the 47th AIAA/ASME/SAE/ASEE Joint Propulsion Conference, San Diego, California, July/August 2011.

[7] Ulbrich, N., "Regression Model Optimization for the Analysis of Experimental Data," AIAA 2009-1344, paper presented at the 47th AIAA Aerospace Sciences Meeting, Orlando, Florida, January 2009.

\section{Glossary}

direct-read format $\equiv$ load format of a balance that can directly be used to compute aerodynamic coefficients in the body axis system; the following loads define the direct-read format for a six-component balance: axial force, normal force, side force, pitching moment, yawing moment, and rolling moment.

moment balance format $\equiv$ load format of a balance consisting of loads that are approximately proportional to related gage outputs; the following loads define the moment balance format for a six-component balance: forward \& aft pitching moment, forward \& aft yawing moment, rolling moment, and axial force.

Iterative Method $\equiv$ load prediction method that first fits the electrical outputs of a balance as a function of the loads and, afterwards, constructs a load iteration scheme from the regression analysis results so that loads can be predicted from outputs during a wind tunnel test; an analyst must make sure that (i) the terms 
of the regression models of the outputs are supported by the calibration data and that (ii) the regression models of the outputs do not have linear or near-linear dependencies.

Non-Iterative Method $\equiv$ load prediction method that directly fits the balance loads as a function of the electrical outputs of a balance; an analyst must make sure that (i) the terms of the regression models of the loads are supported by the calibration data and that (ii) the regression models of the loads do not have linear or near-linear dependencies.

balance moment center $\equiv$ a point on the balance that defines moment arms; the moment arms are needed for the calculation of moments that act of a balance.

tare loads $\equiv$ balance loads that are caused by the weight of the metric part, the calibration body, and other calibration hardware pieces (yoke, rods, weight pans, etc.). 